\title{
10. Heißt krank zu sein sich auch krank zu fühlen? Subjektive Gesundheit und ihr Zusammenhang mit anderen Gesundheitsdimensionen
}

\author{
Svenja M. Spuling, Susanne Wurm, Julia K. Wolff \& Jenna Wünsche
}

\section{Kernaussagen}

Ein Großteil der 40- bis 85-Jährigen bewertet die eigene Gesundheit im Jahr 2014 als gut, wobei jedoch deutliche Alters- und Bildungsunterschiede bestehen: Der Anteil an Personen, die zu einer guten Gesundheitsbewertung kommen, ist unter den 70- bis 85-Jährigen mit 44,6 Prozent im Vergleich zu den 55- bis 69-Jährigen (53,6 Prozent) und den 40- bis 54-Jährigen (64,5 Prozent) am geringsten. Hochgebildete bewerten mit 63,4 Prozent im Vergleich zur mittleren (53,6 Prozent) und niedrigen Bildungsgruppe (36,3 Prozent) am häufigsten ihre Gesundheit als gut.

Nur bei den über 65-Jährigen zeigt sich zwischen 1996 und 2014 ein positiver Wandel der subjektiven Gesundheitsbewertung: Der Anteil von Personen mit guter subjektiver Gesundheitsbewertung ist beispielsweise bei den 66- bis 71-Jährigen zwischen 1996 und 2014 um mehr als zwölf Prozentpunkte angestiegen. Bei den unter 66-Jährigen zeichnet sich dagegen kein vergleichbarer Trend ab.

Die subjektive Gesundheitsbewertung hängt im Jahr 2014 eng mit Erkrankungen, funktionalen Einschränkungen und depressiven Symptomen zusammen: Personen mit guter subjektiver Gesundheitsbewertung geben weniger Erkrankungen, funktionale Einschränkungen und depressive Symptome an. Trotzdem bewertet auch fast die Hälfte der mehrfach erkrankten Personen ihre Gesundheit als gut - gleiches gilt für über 20 Prozent der Personen mit funktionalen Einschränkungen und für etwa jede dritte Person mit mindestens leichten depressiven Symptomen.

Im Jahr 2014 zeigen sich deutliche Bildungsunterschiede in der subjektiven Gesundheitsbewertung bei mehrfach Erkrankten und Personen mit mindestens leichten depressiven Symptomen: Nur ein Viertel der mehrfach erkrankten Personen mit niedriger Bildung berichtet im Jahr 2014 von einer guten subjektive Gesundheit, in der höchsten Bildungsgruppe sind es hingegen mehr als doppelt so viele. Zudem bewerten 17,0 Prozent der niedriggebildeten Personen mit mindestens leichten depressiven Symptomen ihre Gesundheit als gut, wohingegen 37,9 Prozent der hochgebildeten Personen mit mindestens leichten depressiven Symptomen zu einer guten Gesundheitsbewertung kommen. 


\subsection{Einleitung}

Gesundheit ist ein mehrdimensionales Konzept (vgl. Kapitel 8), das nicht nur die körperliche, funktionale und psychische Gesundheit umfasst, sondern auch die subjektive Gesundheitsbewertung berücksichtigt (WHO 2002). Die subjektive Gesundheitsbewertung, das heißt, wie Menschen sich gesundheitlich fühlen und ihre Krankheit(en) und Einschränkungen einschätzen, stellt ein zentrales Maß gesundheitsbezogener Lebensqualität dar und kann eine Reihe anderer Gesundheitsaspekte (zum Beispiel Genesung nach Krankheit) vorhersagen. In besonderem Maße gilt dies für die Sterblichkeits-Vorhersage: Menschen mit einer guten subjektiven Gesundheit leben länger und dies zeigt sich interessanter Weise auch unabhängig von ihrer objektiven Gesundheit (Idler \& Benyamini 1997; Benyamini \& Idler 1999). Darüber hinaus ist bei Menschen mit guter subjektiver Gesundheit die Motivation höher, gesundheitsförderliche Verhaltensweisen auszuführen oder Risikoverhaltensweisen zu verändern (Benyamini 2011).

Bewerten Menschen ihre eigene Gesundheit, so berücksichtigen sie dabei ihren körperlichen Gesundheitszustand und mögliche, damit einhergehende funktionale Einschränkungen. Zusätzlich fließen in die Bewertung aber auch zahlreiche andere Faktoren mit ein, die über das Wissen über den körperlichen Gesundheitszustand hinausgehen - wie zum Beispiel die psychische Gesundheit, das Gesundheitsverhalten oder das Wohlbefinden. Die eigene Einschätzung der Gesundheit spiegelt demnach den individuellen Gesundheitszustand umfassender wider als einzelne Gesundheitsdimensionen oder Diagnosen, da die eigene Lebenssituation und der eigene Lebensstil in die Bewertung einfließen. Es ist daher wichtig, sowohl bei der Gesundheitsberichterstattung als auch in der Gesundheitsversorgung diese Selbsteinschätzung zusätzlich zu den objektiven Gesundheitsmaßen zu berücksichtigen. Gesundheitsscreenings sollten sich deshalb nicht nur auf Laborwerte und diagnostizierte Erkrankungen beziehen, sondern auch die subjektive Gesundheit der Patienten in den Blick nehmen. Da die subjek- tive Einschätzung der Gesundheit nicht nur den aktuellen Gesundheitszustand, sondern auch mögliche gesundheitliche Veränderungen in der Zukunft miteinbezieht (Benyamini 2011), kann eine als schlecht eingeschätzte Gesundheit ein Hinweis auf Risiken sein, die sich (noch) nicht in objektiven gesundheitsbezogenen Maßen widerspiegeln. Insbesondere Personen, die keine diagnostizierte Krankheit haben, ihren Gesundheitszustand aber als schlecht einschätzen, stellen eine Risikogruppe dar: zum einen, da sie möglicherweise medizinisch unterversorgt sind, zum anderen, da sie eine geringe gesundheitsbezogene Lebensqualität haben, die Folgeprobleme (zum Beispiel Fehlzeiten am Arbeitsplatz, psychische Erkrankungen, funktionale Einschränkungen) nach sich ziehen kann.

Im höheren Alter ist es besonders wichtig, verschiedene Gesundheitsdimensionen im $\mathrm{Zu}-$ sammenspiel zu betrachten. Gerade der subjektiven Gesundheit kommt mit steigendem Alter eine wachsende Bedeutung zu. Ein Grund hierfür ist, dass mehreren Studien zufolge die subjektive Gesundheit bis ins hohe Alter im Mittel vergleichsweise stabil bleibt - das heißt, sie nimmt nicht im gleichen Ausmaß ab, wie es die altersbedingte Zunahme an Erkrankungen und funktionalen Einschränkungen (vgl. Kapitel 8) erwarten lassen würde (Leinonen, Heikkinen, \& Jylhä 2001; Spuling, Wiest, Huxhold, \& Wurm 2013). In jüngeren Altersgruppen kommen zwar mehr Menschen zu einer guten subjektiven Gesundheitsbewertung als in älteren Altersgruppen, dennoch ist in bisherigen Studien zu beobachten, dass auch ein großer Teil der älteren Menschen ihre Gesundheit als gut oder sogar sehr gut bewertet.

Auch sind Unterschiede zwischen verschiedenen Bildungsgruppen zu erwarten: Der in früheren Studien konsistent aufgezeigte Befund, dass Bildungsunterschiede zuungunsten Niedriggebildeter hinsichtlich des allgemeinen Gesundheitszustandes bestehen (Ellert \& Kurth 2013; Wurm, Schöllgen, \& Tesch-Römer 2010; Robert Koch-Institut 2015) sollte sich demnach 
auch in der subjektiven Gesundheitsbewertung widerspiegeln.

Unterschiede bei der subjektiven Gesundheitsbewertung von Frauen und Männern waren in den bisherigen Erhebungen des Deutschen Alterssurvey (DEAS) nicht festzustellen (Wurm et al. 2010). Frauen berichten zwar in vielen Studien mehr körperliche Erkrankungen und funktionale Einschränkungen (Robert Koch-Institut 2014), bei der Einschätzung der subjektiven Gesundheit werden jedoch Vergleichsprozesse wirksam, die diese Unterschiede wieder ausgleichen könnten. Es ist anzunehmen, dass sich Frauen bei der Beurteilung ihrer Gesundheit mit anderen Frauen ihres Alters vergleichen. Wenn es nun innerhalb dieser Vergleichsgruppe ,normal' ist, ein bestimmtes Maß an Erkrankungen beziehungsweise Einschränkungen zu haben, dann verlieren diese Kriterien an Wichtigkeit und fließen demnach mit einer geringeren Gewichtung in die subjektive Gesundheitsbewertung hinein.

Neben möglichen Alters-, Bildungs- und Geschlechtsunterschieden soll zudem der Frage nachgegangen werden, inwiefern sich die subjektive Gesundheit über die Erhebungen hinweg gewandelt hat und ob sich dieser Wandel für Personen unterschiedlichen Alters in gleicher Weise gestaltet. Bisherige Befunde zum Wandel der subjektiven Gesundheit sind gemischter $\mathrm{Na}$ tur. Im Vergleich früherer und späterer Geburtsjahrgänge werden sowohl Verbesserungs- als auch Verschlechterungstrends sowie Kontinuität über die Zeit berichtet (Parker \& Thorslund 2007).

Ein zentraler Teil des vorliegenden Kapitels behandelt den Zusammenhang zwischen den verschiedenen Gesundheitsdimensionen mit der subjektiven Gesundheit. Im Mittelpunkt steht dabei die Frage, wie eng ein ,sich gesund fühlen' mit objektiveren Gesundheitsmaßen verbunden ist, also einer geringen Zahl von selbstberichteten Erkrankungen, funktionalen Einschränkungen und depressiven Symptomen - und umgekehrt, wie viele Menschen, die von Krankheiten, funktionalen Einschränkungen und depressiven Symptomen berichten, sich dennoch subjektiv gesund fühlen.

Der Zusammenhang von Erkrankungen, funktionalen Einschränkungen und depressiven Symptomen mit der subjektiven Gesundheit kann sich dabei für Personen mit verschiedener wirtschaftlicher, sozialer oder psychologischer Ressourcenausstattung unterscheiden. Die Bildung spielt dabei eine wichtige Rolle: zum einen, weil sie selbst eine relevante Gesundheitsressource darstellt, zum anderen, weil sie mit der Aktivierung weiterer Ressourcen, wie beispielsweise sozialer Unterstützung, einer optimistischen und hoffnungsvollen Zukunftsperspektive sowie günstigen Gesundheitsverhaltensweisen, in Verbindung steht (Gallo \& Matthews 2003; Schöllgen, Huxhold, Schüz, \& Tesch-Römer 2011). Demnach müssten sich Höhergebildete aufgrund ihres doppelten Ressourcenvorteils besser an die negativen Auswirkungen einer schlechten Gesundheit anpassen können als niedriger gebildete Personen. Dies sollte dazu führen, dass der Zusammenhang zwischen den verschiedenen Gesundheitsdimensionen und der subjektiven Gesundheit in höheren Bildungsgruppen schwächer ausfällt. In anderen Worten: Bei einem gleichermaßen schlechten körperlichen Gesundheitszustand sollten Personen mit einer höheren Bildung häufiger zu einer guten Gesundheitsbewertung kommen als niedriggebildete Personen.

Zusammenfassend werden im vorliegenden Kapitel die folgenden Forschungsfragen untersucht:

1. Wie ist die subjektive Gesundheit im Jahr 2014 und wie hat sie sich seit 1996 gewandelt?

2. Wie hängt die subjektive Gesundheit mit anderen Gesundheitsdimensionen zusammen?

3. Unterscheidet sich der Zusammenhang zwischen der subjektiven Gesundheit und anderen Gesundheitsdimensionen für Personen aus unterschiedlichen Bildungsgruppen? 


\subsection{Datengrundlage ${ }^{1}$}

Daten. Im vorliegenden Kapitel werden die Daten der 40- bis 85-Jährigen aller vier Erhebungen des DEAS (1996, 2002, 2008 und 2014) verwendet. Der Schwerpunkt der Analysen nutzt jedoch ausschließlich die Daten von 2014, um Zusammenhänge der Gesundheitsdimensionen untereinander $\mathrm{zu}$ untersuchen. Subjektive und funktionale Gesundheit sowie depressive Symptome wurden im Rahmen des persönlichen Interviews erfasst. Selbstberichtete Erkrankungen wurden im schriftlichen Fragebogen erhoben.

Subjektive Gesundheit. Alle Befragten gaben an, wie sie ihren derzeitigen Gesundheitszustand bewerten. Die möglichen Antwortalternativen lauteten dabei: ,sehr gut', ,gut', ,mittel', ,schlecht' und ,sehr schlecht. Die Antworten zu sehr guter oder guter Gesundheit wurden in eine Gruppe zusammengefasst, die im Folgenden als Personen mit guter subjektiver Gesundheit bezeichnet wird, und die Antworten zu schlechter und sehr schlechter Gesundheit wurden in eine Gruppe zusammengefasst, die im Folgenden als Personen mit schlechter subjektiver Gesundheit bezeichnet wird. Für die deskriptiven Ergebnisse zur subjektiven Gesundheit nach den Gruppierungsvariablen werden alle drei Gruppen der subjektiven Gesundheit (gut, mittel, schlecht) berichtet. Gleiches gilt für die Betrachtung des Wandels in der subjektiven Gesundheit. Bei den Zusammenhangsanalysen mit den anderen Gesundheitsdimensionen werden nur die beiden Gruppen mit guter sowie schlechter Gesundheitsbewertung betrachtet.

Selbstberichtete Erkrankungen. Für die selbstberichteten Erkrankungen wird derselbe Indikator wie in Kapitel 8 verwendet. Es wird der Anteil der Personen dargestellt, die keine oder eine Erkrankung angeben und mit jenen verglichen, die zwei und mehr Erkrankungen haben.

Funktionale Gesundheit. Analog zu Kapitel 8, werden Personen, die keine bis geringe funktionale Einschränkungen nennen und damit von einer guten funktionale Gesundheit berichten (Mittelwert kleiner gleich 1,3 auf der Subska-

1 Die Daten des DEAS können für wissenschaftliche Zwecke kostenlos beim Forschungsdatenzentrum des DZA (www.fdz-dza.de) bezogen werden. la körperliche Funktionsfähigkeit des SF-36) mit Personen verglichen, bei denen mittlere bis schwere funktionale Einschränkungen vorhanden sind (Mittelwert größer als 1,3).

Depressive Symptome. Das verwendete Maß für die depressiven Symptome wird in Kapitel 11 ausführlicher beschrieben. Der Anteil an Personen, die keine depressiven Symptome berichten (Summenwert kleiner gleich 8 auf der Kurzform der ADS-K-Skala - Kurzfassung der Allgemeinen Depressionsskala) wird mit denen verglichen, die mindestens leichte depressive Symptome berichten (Summenwert größer als 8).

Gruppierungsvariablen. Um Altersgruppenunterschiede $\mathrm{zu}$ veranschaulichen, werden drei Altersgruppen abgebildet ( 40 bis 54 Jahre, 55 bis 69 Jahre und 70 bis 85 Jahre). Zur Untersuchung des Wandels in der subjektiven Gesundheit wird eine feinere Altersgruppierungsvariable mit sieben Kategorien verwendet ( 42 bis 47 Jahre, 48 bis 53 Jahre, 54 bis 59 Jahre, 60 bis 65 Jahre, 66 bis 71 Jahre, 72 bis 77 Jahre, 78 bis 83 Jahre). Dies soll sicherstellen, dass es bei der Untersuchung von Geburtskohorteneffekten zu keinen Überschneidungen zwischen Erhebungszeitpunkten und Altersgruppen kommt (vgl. Kapitel 2). Zur Beschreibung von Bildungsunterschieden wurden - basierend auf einer reduzierten ISCED-Klassifizierung (International Standard Classification of Education, UNESCO 2012) - drei Bildungsgruppen unterschieden: Niedrig-, Mittel- und Hochgebildete (vgl. Kapitel 2). Weiterhin wird die subjektive Gesundheit von Männern und Frauen betrachtet.

Analysen. Signifikanztestungen zur statistischen Absicherung der Gruppenunterschiede erfolgten mit Hilfe von (ordinalen) logistischen Regressionen, wobei für die Stratifizierungsvariablen Altersgruppe, Geschlecht und Region (Ost-/Westdeutschland) kontrolliert wurde. In den Abbildungen unter Abschnitt 10.3 werden gewichtete Häufigkeiten dargestellt, wohingegen die Prozentzahlen in den Zusammenhangsanalysen unter 10.4 und 10.5 direkt aus den am Mikrozensus gewichteten logistischen Regressionen errechnet wurden. Das genaue Vorgehen ist in Kapitel 2 beschrieben. 


\subsection{Subjektive Gesundheit im Jahr 2014 und im Wandel}

Im Folgenden werden die Ergebnisse zu den Unterschieden in der subjektiven Gesundheit zwischen den gesellschaftlichen Gruppen dargestellt. Im Anschluss werden die Befunde zum Wandel in der subjektiven Gesundheitsbewertung zwischen 1996 und 2014 beschrieben.

\section{Ein Großteil der 40- bis 85-Jährigen bewertet die eigene Gesundheit im Jahr 2014 als gut, wobei jedoch deutliche Alters- und Bildungsunterschiede bestehen.}

Über die Hälfte der 40- bis 85-Jährigen schätzt die eigene Gesundheit als gut ein (55,8 Prozent). Nur 11,3 Prozent bewerten ihre Gesundheit als schlecht und 32,9 Prozent als mittel (Abbildung 10-1). Wie erwartet, zeigen sich hinsichtlich der subjektiven Gesundheit keine Unterschiede zwischen Frauen und Männern, jedoch deutliche Unterschiede zwischen den Alters- und Bildungsgruppen (Abbildung 10-1).

In der jüngsten untersuchten Altersgruppe, den 40 - bis 54-Jährigen, schätzen knapp zwei Drittel (64,5 Prozent) ihre Gesundheit als gut ein. Dieser Anteil ist bei den 55- bis 69-Jährigen (um 10,9 Prozentpunkte) und bei den 70- bis 85-Jährigen (um 19,9 Prozentpunkte) geringer, das heißt die subjektive Gesundheit wird in höheren Altersgruppen als schlechter eingeschätzt. Trotzdem bewerten auch in der Altersgruppe der 70- bis 85-Jährigen noch 44,6 Prozent ihre Gesundheit als gut. Dementsprechend liegt der
Anteil an Personen mit einer schlechten Gesundheitsbewertung bei 8,7 Prozent unter den 40- bis 54-Jährigen, bei 12,0 Prozent unter den 55- bis 69-Jährigen und bei 14,6 Prozent unter den 70- bis 85-Jährigen. Doch auch in der höchsten Altersgruppe bewerten noch über 85 Prozent ihren Gesundheitszustand als gut oder mittel, wobei der Anteil an Personen mit einer mittleren subjektiven Gesundheit bei den 70- bis 85-Jährigen im Vergleich zu den beiden anderen Altersgruppen auf dem höchsten Niveau liegt (40,9 Prozent). Folglich kommen ältere Personen zwar im Vergleich zu jüngeren zu einer weniger positiven Bewertung ihrer Gesundheit, ein großer Teil schätzt seine Gesundheit aber selbst im hohen Alter noch als gut ein.

Es finden sich auch die erwarteten Unterschiede zwischen den Bildungsgruppen. Personen mit höherer Bildung bewerten demnach ihre Gesundheit besser als Personen mit niedriger Bildung. Nur etwas mehr als ein Drittel der Personen mit niedriger Bildung (36,3 Prozent), aber knapp zwei Drittel der Personen mit höherer Bildung (63,4 Prozent) kommen zu einer guten Gesundheitsbewertung. Der Anteil an Personen mit schlechter subjektiver Gesundheit liegt in der niedrigen Bildungsgruppe bei 21,5 Prozent, während er nur 6,7 Prozent in der höchsten Bildungsgruppe beträgt. Eine mittlere Gesundheitsbewertung ist zudem innerhalb der niedrigen Bildungsgruppe (42,1 Prozent) am meisten verbreitet und am seltensten bei den Hochgebildeten (29,9 Prozent). 
Abbildung 10-1: Subjektive Gesundheit, gesamt, nach Alter, Geschlecht und Bildung, im Jahr 2014 (in Prozent)

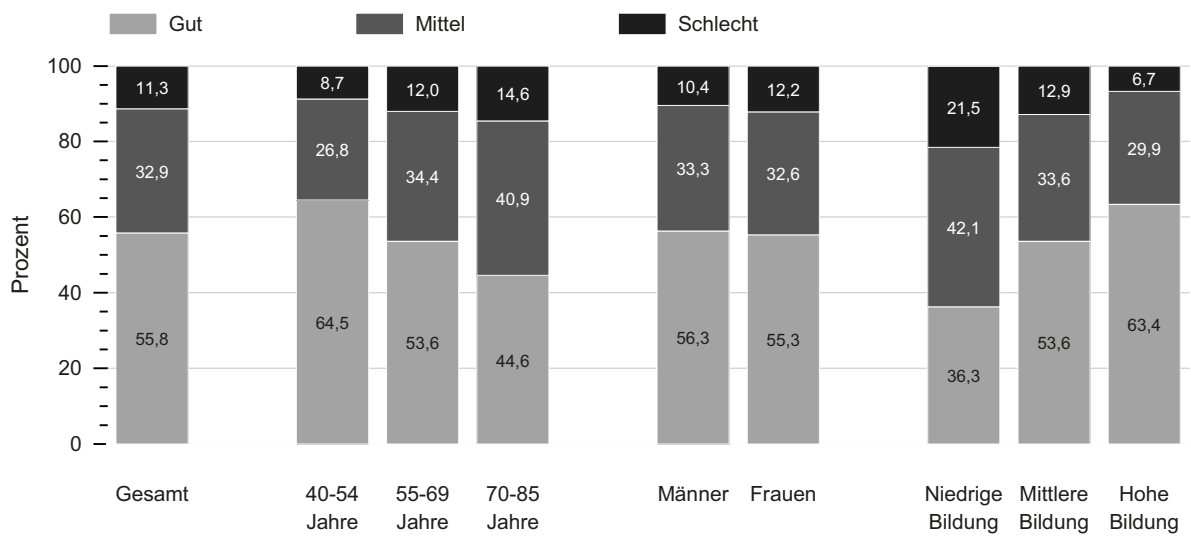

Quelle: DEAS 2014 ( $n=5.994)$, gewichtet, gerundete Angaben; $(p<, 05)$.

Alters- und Bildungsunterschiede in allen drei Kategorien der subjektiven Gesundheit signifikant. Geschlechtsunterschiede in allen drei Kategorien der subjektiven Gesundheit nicht signifikant.

\section{Nur bei den über 65-Jährigen zeigt sich zwischen 1996 und 2014 ein positiver Wandel der subjektiven Gesundheitsbewertung.}

Ein Vergleich der subjektiven Gesundheit über die Erhebungsjahre hinweg macht deutlich, dass die 40- bis 85 -Jährigen ihre Gesundheit in den Jahren 2002, 2008 und 2014 häufiger als gut und seltener als schlecht bewertet haben als im ersten Erhebungsjahr 1996 (vgl. Tabelle A 10-1 im Anhang).

Ein separater Blick auf die Entwicklung innerhalb der verschiedenen Altersgruppen (Abbildung 10-2) zeigt jedoch, dass sich der positive Wandel in der subjektiven Gesundheit auf die höheren Altersgruppen (ab 66 Jahren) begrenzt: Die 66- bis 71-Jährigen haben einen kontinuierlichen Anstieg im Anteil der Personen verzeichnet, die ihre Gesundheit als gut bewerten. Im Jahr 2014 liegt dieser Anteil mit 58,1 Prozent auf einem signifikant höheren Niveau als in den Jahren 2008 (51,7 Prozent), 2002 (49,8 Prozent) und 1996 (45,7 Prozent). Auch die 72- bis 77-Jährigen geben im Jahr 2014 häufiger einen guten beziehungsweise seltener einen schlech- ten Gesundheitszustand an als gleichaltrige Personen im Jahr 1996. In der ältesten Altersgruppe (78- bis 83-Jährige) lässt sich die positive Entwicklung erst seit 2002 beobachten: Der Anteil an Personen, die ihre Gesundheit als gut bewerten, ist innerhalb dieser Altersgruppe zwischen 2002 und 2014 um 8,1 Prozentpunkte (von 29,7 Prozent auf 37,8 Prozent) gestiegen, während der Anteil an Personen, die ihre Gesundheit als schlecht bewerten um 14,7 Prozentpunkte (von 32,3 Prozent auf 17,6 Prozent) gesunken ist.

Bei den jüngeren Personen ergibt sich dagegen ein weniger eindeutiges Bild für den Wandel der subjektiven Gesundheit. Dieses reicht von einer Stabilität in der Gesundheitseinschätzung bei den 48 - bis 53-Jährigen und 60- bis 65-Jährigen, über eine wechselhafte Entwicklung bei den 54bis 59-Jährigen, bis hin zu einer durchgängigen Umkehrung des positiven Trends in der jüngsten Altersgruppe: Die 42- bis 47-Jährigen im Jahr 2014 fühlen sich weniger gesund als Personen, die 1996 im gleichen Alter waren. Die Ergebnisse zeigen also, dass sich die subjektive Gesundheit zwischen 1996 und 2014 uneinheitlich, aber zugunsten älterer Altersgruppen entwickelt hat. 
Abbildung 10-2: Subjektive Gesundheit, nach Alter, in den Jahren 1996, 2002, 2008 und 2014 (in Prozent)

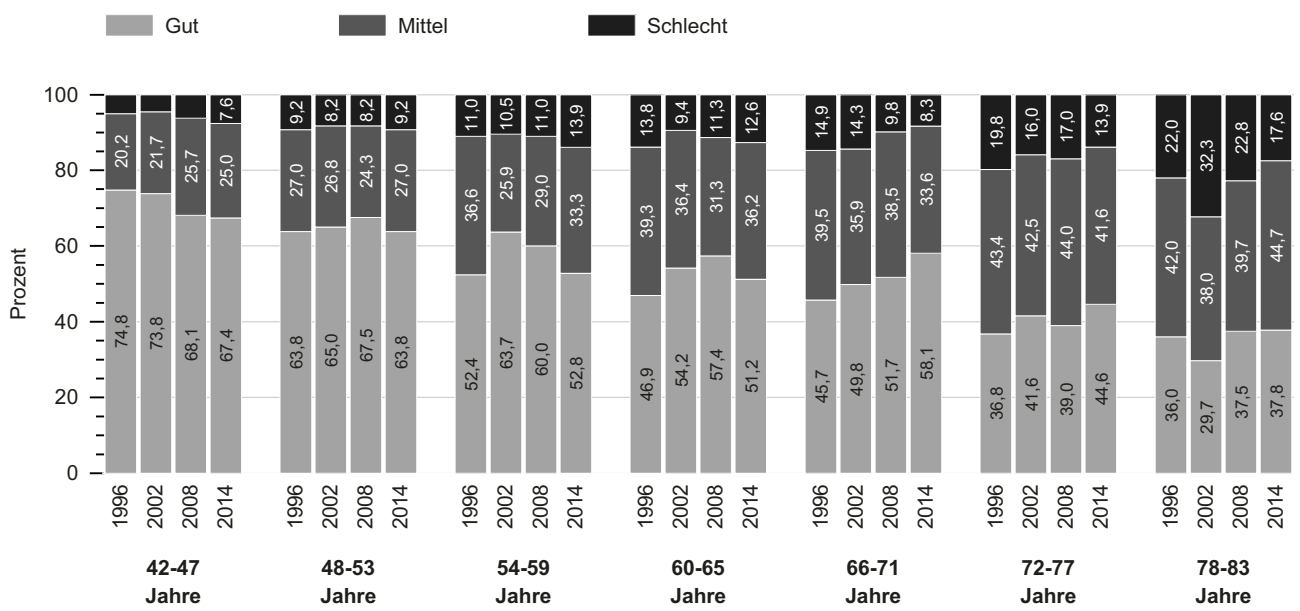

$\overline{\text { Quelle: DEAS } 1996}(n=4.833), 2002(n=3.081), 2008(n=6.195), 2014(n=5.994)$, gewichtet, gerundete Angaben; $(p<, 05)$.

Interaktion zwischen Alter und Erhebungsjahr ist signifikant. Signifikante Zunahme in der Kategorie ,gut' und signifikante Abnahme in der Kategorie ,schlecht' zwischen 2002 und 2014 bei den 78- bis 83-Jährigen beziehungsweise zwischen 1996 und 2014 bei den 66- bis 77-Jährigen. Signifikante Abnahme in der Kategorie ,gut' und signifikante Zunahme in der Kategorie ,schlecht' von 1996 bis 2014 bei den 42- bis 47-Jährigen. Signifikante Zunahme zwischen 1996 und 2002 und signifikante Abnahme zwischen 2002 und 2014 in der Kategorie ,gut' bei den 54- bis 59-Jährigen. Stabilität zwischen 1996 und 2014 in allen Kategorien bei den 48- bis 53-Jährigen und 60- bis 65-Jährigen.

\subsection{Zusammenhang zwischen Erkrankungen, funktionalen Einschränkungen und depressiven Symptomen mit der subjektiven Gesundheitsbewertung}

Die subjektive Gesundheit sollte allerdings nicht nur allein betrachtet werden. Auch der Zusammenhang zwischen der subjektiven Einschätzung des Gesundheitszustandes und anderen Gesundheitsindikatoren ist von Interesse.

\section{Die subjektive Gesundheitsbewertung hängt im Jahr 2014 eng mit Erkrankungen, funktionalen Einschränkungen und depressiven Symptomen zusammen.}

Personen, die kaum Erkrankungen, funktionale Einschränkungen beziehungsweise depressive
Symptome berichten, bewerten auch ihren allgemeinen Gesundheitszustand besser.

Abbildung 10-3 veranschaulicht den Zusammenhang zwischen den verschiedenen Gesundheitsdimensionen und der subjektiven Gesundheit. Dabei fällt auf, dass zwar eine deutliche Mehrheit der Personen, die keine oder nur eine Erkrankung berichtet, auch zu einer guten Gesundheitseinschätzung kommt, dass aber auch fast die Hälfte der mehrfach erkrankten Personen ihre Gesundheit als gut bewertet. Während nur 3,5 Prozent der 40- bis 85-Jährigen mit keiner oder einer Erkrankung ihre Gesundheit als schlecht bewerten, steigt dieser Anteil auf 15,0 Prozent in der Gruppe der Personen mit 
zwei oder mehr Erkrankungen. Trotzdem ist bei mehrfach erkrankten Personen der Anteil mit einer guten Gesundheitsbewertung immer noch dreimal so groß wie der Anteil mit einer schlechten Gesundheitsbewertung.

Ein ähnliches Bild zeigt sich auch für den Zusammenhang zwischen der subjektiven Gesundheit und dem Vorhandensein funktionaler Einschränkungen beziehungsweise depressiver Symptome. Ein großer Anteil der Personen, der keine bis geringe Einschränkungen oder keine depressiven Symptome berichtet, schätzt auch die subjektive Gesundheit erwartungsgemäß als gut ein. Bemerkenswert ist, dass auch ein nennenswerter Anteil an Personen mit einer einge- schränkten funktionalen Gesundheit $\mathrm{zu}$ einer guten subjektiven Gesundheitseinschätzung kommt. So bewerten auch 21,6 Prozent der Personen, die mittlere bis schwere funktionale Einschränkungen berichten, und 28,2 Prozent der Personen mit mindestens leichten depressiven Symptomen ihre Gesundheit als gut.

Es lassen sich also bedeutsame Zusammenhänge zwischen der subjektiven Gesundheitsbewertung und anderen Gesundheitsdimensionen beobachten, wobei viele Personen selbst bei Vorhandensein von gesundheitlichen Beeinträchtigungen $\mathrm{zu}$ einer positiven Gesundheitsbewertung kommen.

Abbildung 10-3: Anteile der Personen mit guter und schlechter subjektiver Gesundheit nach selbstberichteten Erkrankungen, funktionalen Einschränkungen und depressiven Symptomen, im Jahr 2014 (in Prozent)

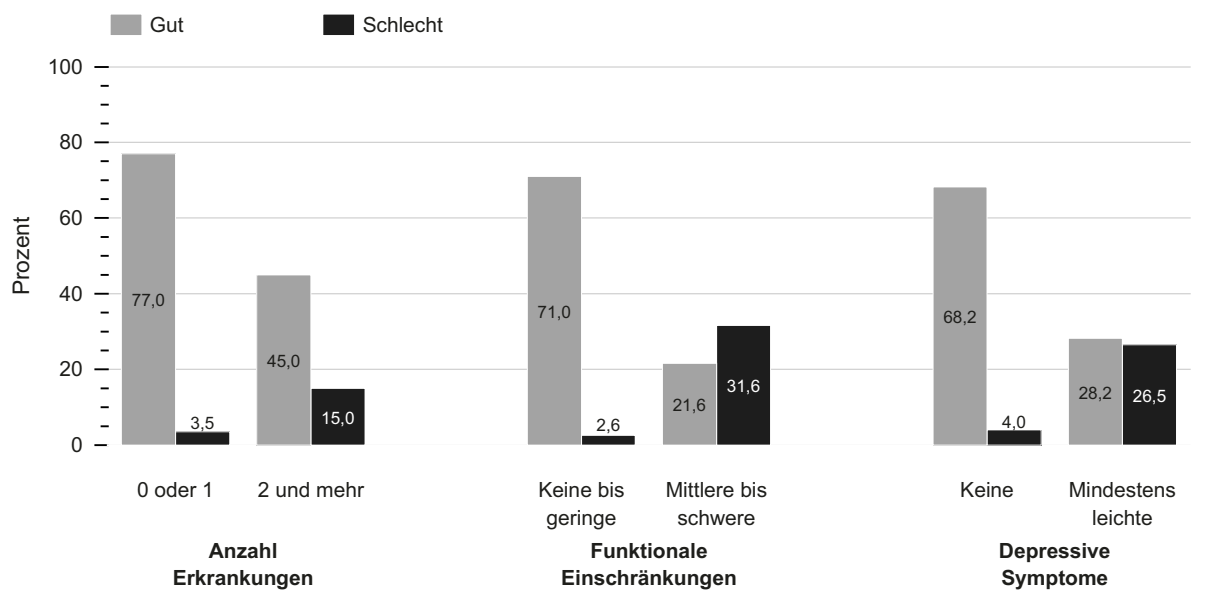

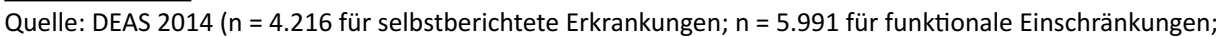
$\mathrm{n}=5.852$ für depressive Symptome), gewichtet, gerundete Angaben; $(p<, 05)$.

Der Zusammenhang zwischen subjektiver Gesundheit und selbstberichteten Erkrankungen, funktionalen Einschränkungen sowie depressiven Symptomen ist signifikant. Signifikante Unterschiede zwischen den Ausprägungen von Erkrankungen, Einschränkungen bzw. depressiven Symptomen sowohl in der Kategorie ,gut' als auch in der Kategorie, schlecht'. 


\subsection{Bildungsunterschiede im Zusammenhang zwischen Erkrankungen, funktionalen Einschränkungen und depressiven Symptomen mit der subjektiven Gesundheitsbewertung}

Die subjektive Gesundheit hängt also mit anderen Dimensionen der Gesundheit zusammen. Darüber hinaus scheint das Bildungsniveau Einfluss darauf zu haben, wie sich diese Zusammenhänge gestalten.

\section{Im Jahr 2014 zeigen sich deutliche Bildungsunterschiede in der subjektiven Gesundheitsbewertung bei mehrfach Erkrankten und Personen mit mindestens leichten depressiven Symptomen.}

Der Zusammenhang zwischen subjektiver Gesundheit und selbstberichteten Erkrankungen ist bei hoher Bildung am geringsten (Abbildung 10-4 a). Zwischen Personen, die null oder eine Erkrankung angeben, lassen sich zwar keine Bildungsunterschiede im Hinblick auf eine gute Gesundheitsbewertung beobachten, doch bei Vorhandensein von Mehrfacherkrankungen wird der Bildungsgradient sichtbar: Im Jahr 2014 ist der Anteil multimorbider Personen mit einer guten subjektiven Gesundheitsbewertung innerhalb der höchsten Bildungsgruppe mit 53,4 Prozent mehr als doppelt so groß wie in der niedrigsten Bildungsgruppe (25,0 Prozent). Demnach gelingt es Hochgebildeten im Vergleich zu Personen mit mittlerer und niedriger
Bildung am ehesten trotz Mehrfacherkrankungen eine gute subjektive Gesundheit aufrechtzuerhalten.

Ein ähnliches Bild ist für den bildungsabhängigen Zusammenhang zwischen dem Vorhandensein depressiver Symptome und der subjektiven Gesundheit zu erkennen (Abbildung 10-4 c). Auch hier zeigt sich in der höchsten Bildungsgruppe der schwächste Zusammenhang zwischen depressiven Symptomen und subjektiver Gesundheit. Anhand von Abbildung 10-4 c wird erkenntlich, dass höhere Bildungsgruppen generell eine bessere Gesundheitsbewertung haben. Besonders deutlich zeigen sich die Bildungsunterschiede aber für jene, die mindestens leichte depressive Symptome berichten. Hier fallen die Bildungsunterschiede stärker und zuungunsten niedriggebildeter Personen aus.

Für den Zusammenhang zwischen funktionalen Einschränkungen und der subjektiven Gesundheitsbewertung finden sich hingegen keine statistisch bedeutsamen Unterschiede zwischen den Bildungsgruppen (Abbildung 10-4 b). Bei Personen mit niedrigem, mittlerem und hohem Bildungsabschluss spiegeln sich Unterschiede im funktionalen Gesundheitsstatus gleichermaßen in der subjektiven Gesundheitsbewertung wider. 
166 | Subjektive Gesundheit und ihr Zusammenhang mit anderen Gesundheitsdimensionen

Abbildung 10-4: Anteile der Personen mit guter subjektiver Gesundheit nach selbstberichteten Erkrankungen, funktionalen Einschränkungen und depressiven Symptomen, nach Bildung, 2014 (in Prozent)

a) Selbstberichtete Erkrankungen

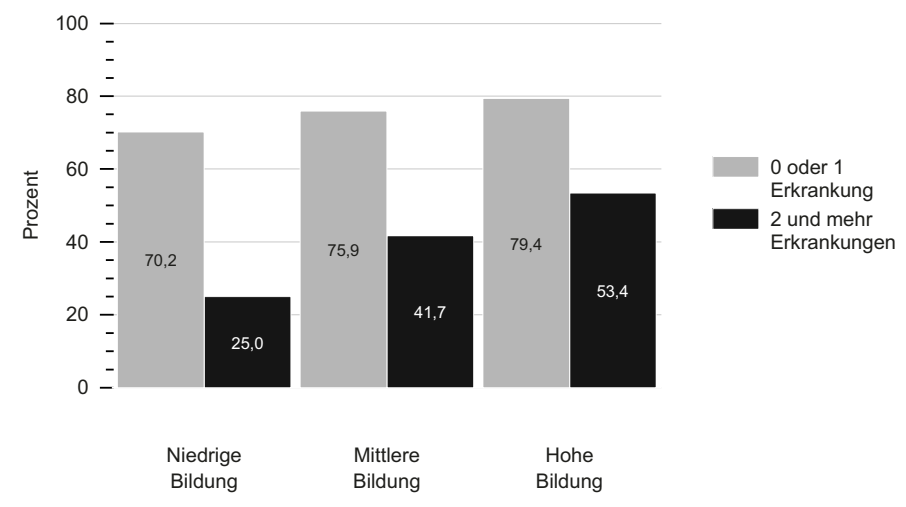

b) Funktionale Einschränkungen

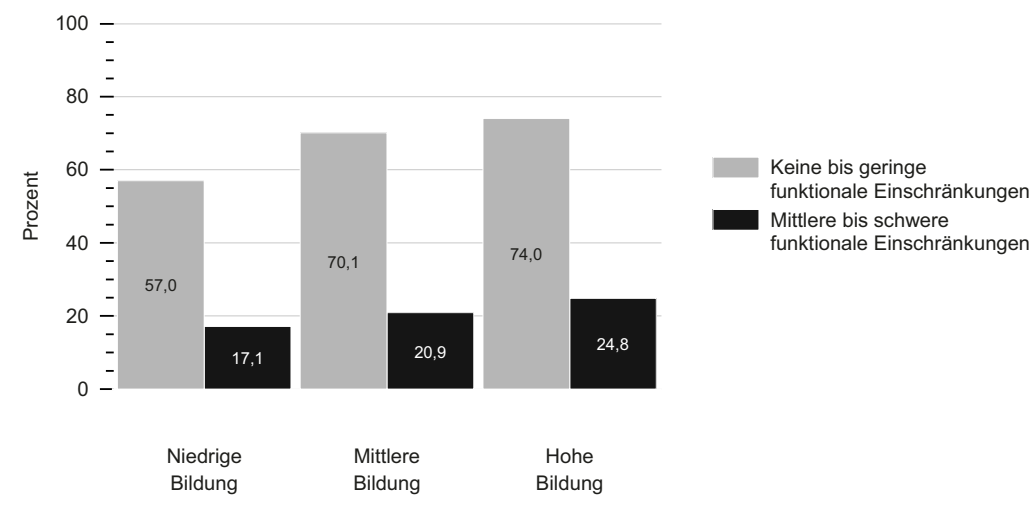




\section{c) Depressive Symptome}

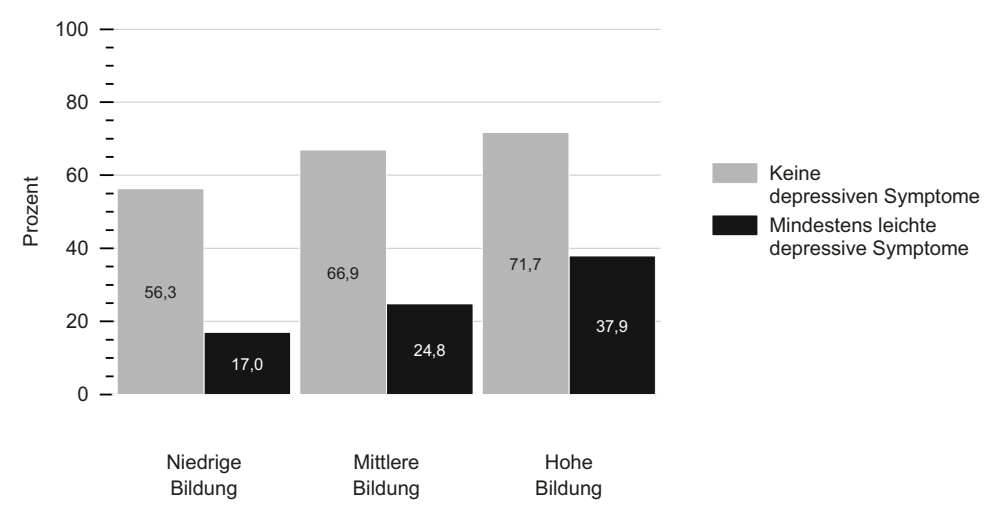

Quelle: DEAS 2014 ( $n=4.215$ für selbstberichtete Erkrankungen; $n=5.990$ für funktionale Einschränkungen; $\mathrm{n}=5.851$ für depressive Symptome), gewichtet, gerundete Angaben; $(p<, 05)$.

Signifikante Interaktion zwischen selbstberichteten Erkrankungen und Bildung sowie zwischen depressiven Symptomen und Bildung. Für Personen mit höherer Bildung ist der Zusammenhang schwächer (geringere Unterschiede zwischen den beiden Ausprägungen von Erkrankungen bzw. depressiven Symptomen bei höher Gebildeten). Der Zusammenhang zwischen funktionalen Einschränkungen und subjektiver Gesundheit unterscheidet sich nicht nach Bildungsgruppe; die Interaktion ist nicht signifikant.

\subsection{Diskussion und Implikationen}

Die subjektive Gesundheit ist ein zentrales Maß gesundheitsbezogener Lebensqualität und ein bedeutsamer Indikator für Langlebigkeit. Die Werte subjektiver Gesundheit reflektieren demnach, wie gut es Menschen gesundheitlich geht und liefern zugleich Anhaltspunkte dafür, wie gut es einer Gesellschaft geht (vgl. Kapitel 11). Da die subjektive Gesundheit nicht allein von objektiven Gesundheitsindikatoren abhängig ist, sondern auch durch die individuelle Lebenssituation, den eigenen Lebensstil und durch Erwartungen im Hinblick auf zukünftige gesundheitliche Veränderungen geprägt ist, müssen objektive und subjektive Gesundheitseinschätzungen nicht zwangsläufig übereinstimmen. Einige Menschen bewerten ihre Gesundheit trotz eines objektiv guten Gesundheitszustandes als schlecht (z. B. Hong, Zarit, \& Malmberg 2004). Solche Inkongruenzen deuten darauf hin, dass manche gesundheitliche Beeinträchtigungen und Risiken durch die bloße Diagnostik von Erkrankungen nicht greifbar werden. Um einer
Unterschätzung der gesundheitlichen Belastung von Personen vorzubeugen, sollte die subjektive Gesundheitsbewertung deshalb nicht nur in der Gesundheitsberichterstattung, sondern auch bei der gesundheitlichen Vorsorge und im Rahmen von medizinischen Behandlungen Beachtung finden.

Erfreulicherweise bewertet ein großer Teil der Menschen im Alter zwischen 40 und 85 Jahren die eigene Gesundheit im Jahr 2014 positiv. Diese subjektive Gesundheitsbewertung wird unter anderem von den Erkrankungen, funktionalen Einschränkungen und depressiven Symptomen einer Person beeinflusst. Doch selbst bei Vorliegen gesundheitlicher Einschränkungen, gibt noch jede fünfte bis fast jede zweite Person an, eine gute Gesundheit zu haben. Daher ist ein zentrales Ergebnis dieses Kapitels, dass eine gute subjektive Bewertung der Gesundheit auch angesichts von Beeinträchtigungen möglich ist. Dies ist auf Anpassungsprozesse zurückzuführen (Leinonen, Heikkinen, \& Jylhä 2002), die 
jedoch in manchen gesellschaftlichen Gruppen aufgrund von größeren Ressourcen besser gelingen als in anderen.

Dieser Einfluss der Ressourcenausstattung zeigt sich in einem klaren Bildungsgradienten: Niedriger Gebildete bewerten ihre Gesundheit deutlich seltener als gut als Höhergebildete. Neben dem schlechteren Gesundheitszustand (vgl. Kapitel 8) bringen Niedriggebildete weniger soziale Ressourcen mit (vgl. Kapitel 17), um gesundheitliche Einschränkungen zu bewältigen. Hinzu kommt, dass sich bei Personen mit einem niedrigen Bildungsabschluss in vielerlei Hinsicht ein weniger vorteilhaftes Gesundheitsverhalten beobachten lässt (vgl. Kapitel 9). Diese Faktoren können sich in einer schlechteren Bewertung der Gesundheit niederschlagen. Die Bildungsunterschiede zeigen sich aber auch im Zusammenhang der subjektiven Gesundheit mit den anderen Gesundheitsindikatoren, zumindest im Hinblick auf Erkrankungen und depressive Symptome. Höher Gebildete geben trotz mehrerer Erkrankungen und mindestens leichter depressiver Symptome eher eine gute subjektive Gesundheit an als niedriggebildete Personen. Bildung spielte jedoch keine bedeutsame Rolle für den Zusammenhang zwischen funktionalen Einschränkungen und subjektiver Gesundheit, was darauf zurückzuführen sein könnte, dass mit Blick auf die funktionale Gesundheit eher das Einkommen ausschlaggebend ist (vgl. auch Schöllgen et al. 2011): Bildung kann funktionale Einschränkungen nicht direkt kompensieren, das Einkommen kann dies eher leisten, etwa in der Form, dass Unterstützungen, wie zum Beispiel durch eine Haushaltshilfe, technische Hilfsmittel oder Wohnraumanpassungen, eher in Anspruch genommen werden können.

Auch im späteren Lebensverlauf scheint die Anpassung an eine altersbedingte Verschlechterung der Gesundheit irgendwann an ihre Grenzen stoßen: So kommt es in älteren Altersgruppen zu einer Abnahme des Anteils an Personen, die ihre Gesundheit als gut bewerten, und gleichzeitig zu einer Zunahme des Anteils an Personen, die ihre Gesundheit als schlecht bewerten.

Im Wandel zeigt sich allerdings ein positiver Trend für die Menschen im Ruhestandsalter: Innerhalb der höheren Altersgruppen ab 66 Jahren zeichnet sich seit 1996, beziehungsweise seit 2002 für die Gruppe der 78- bis 83-Jährigen, eine positive Entwicklung in Richtung einer besseren Gesundheitsbewertung ab. Diese Entwicklung steht in Einklang mit den Ergebnissen des Robert Koch-Instituts von 2010 (Robert Koch-Institut 2010) und weist außerdem Parallelen zu den DEAS-Befunden in Bezug auf den Wandel der funktionalen Gesundheit (vgl. Kapitel 8) und der Depressivität (vgl. Kapitel 11) auf. Da die subjektive Gesundheit sowohl mit funktionalen Einschränkungen als auch mit depressiven Symptomen zusammenhängt, deutet einiges darauf hin, dass diese Entwicklungen in Bezug zueinander stehen. Der Trend hin zu einer besseren subjektiven Gesundheitsbewertung bei den über 65-Jährigen ist besonders vor dem Hintergrund bemerkenswert, dass sich im DEAS zwischen 2008 und 2014 eine Zunahme im Anteil der Personen mit Mehrfacherkrankungen abgezeichnet hat, von der alle Altersgruppen gleichermaßen betroffen sind (vgl. Kapitel 8).

Im mittleren Erwachsenenalter kann dieser positive Wandel dagegen nicht beobachtet werden. Die Verschlechterung der subjektiven Gesundheit in der jüngsten Altersgruppe könnte nicht nur einen objektiv schlechteren Gesundheitszustand widerspiegeln, sondern auch dadurch zustande gekommen sein, dass nachrückende Generationen ein anderes Verständnis von Gesundheit haben beziehungsweise höhere Ansprüche an sie stellen. Deshalb fallen möglicherweise bereits geringe Beeinträchtigungen bei der Beurteilung des aktuellen Gesundheitszustandes stärker ins Gewicht (Parker \& Thorslund 2007). Das Ausbleiben einer Verbesserung der subjektiven Gesundheit bei den unter 66-Jährigen könnte jedoch ebenfalls mit den ungünstigen Entwicklungen in den selbstberichteten Erkrankungen, der funktionalen Gesundheit und der Depressivität innerhalb dieser Altersgruppen zusammenhängen (vgl. Kapitel 8 und 11). Alles in allem deuten die Ergebnisse darauf hin, dass sich die gesundheitliche Belastung für Personen im Erwerbsalter über die Zeit eher vergrößert hat. Da aber neben den objektiven Aspekten des Gesundheitszustandes auch die Ressourcenausstattung einer Person sowie sich wandelnde Vergleichsstandards und Umweltfaktoren in die 
subjektive Gesundheitsbewertung mit einfließen, kann man vom Wandel der subjektiven Gesundheit nicht ausschließlich auf einen Wandel des Gesundheitszustandes schließen.

Insgesamt weisen die Ergebnisse darauf hin, dass vor allem bei einer subjektiv als schlecht bewerteten Gesundheit Handlungsbedarf besteht. Demnach sollte niedriggebildeten und älteren Personen besondere Aufmerksamkeit bei der Gesundheitsförderung geschenkt werden. Weiterhin ist es sinnvoll, Ressourcen (zum Beispiel Gesundheitsverhaltensweisen und Ge- sundheitswissen) zu fördern, die die negativen Auswirkungen von gesundheitlichen Beeinträchtigungen abschwächen. Auf diesem Weg könnte die Lebensqualität von Personen - auch bei dauerhaften gesundheitlichen Einschränkungen - entschieden verbessert werden. Die Werte der subjektiven Gesundheit liefern in diesem Zusammenhang einen hilfreichen Indikator dafür, wie wirksam ressourcenfördernde Präventionsmaßnahmen zur Bewältigung von Erkrankungen und Beeinträchtigungen sind.

\section{Literatur}

Benyamini, Y. (2011). Why does self-rated health predict mortality? An update on current knowledge and a research agenda for psychologists. Psychology and Health, 26(11), 1407-1413. doi: 10.1080/08870446.2011.621703.

Benyamini, Y., \& Idler, E. L. (1999). Community studies reporting association between self-rated health and mortality. Research on Aging, 21(3), 392-401. doi: 10.1177/0164027599213002.

Ellert, J., \& Kurth, B. M. (2013). Gesundheitsbezogene Lebensqualität bei Erwachsenen in Deutschland. Ergebnisse der Studie zur Gesundheit Erwachsener in Deutschland (DEGS1). Bundesgesundheitsblatt, 56, 643-649. doi: 10.1007/s00103-013-1700-y.

Gallo, L. C., \& Matthews, K. A. (2003). Understanding the association between socioeconomic status and physical health: Do negative emotions play a role? Psychological Bulletin, 129(1), 10-51. doi: 10.1037/00332909.129.1.10.

Hong, T. B., Zarit, S. H., \& Malmberg, B. (2004). The role of health congruence in functional status and depression. The Journals of Gerontology Series B: Psychological Sciences and Social Sciences, 59(4), P151-P157. doi: 10.1093/geronb/59.4.P151.

Idler, E. L., \& Benyamini, Y. (1997). Self-rated health and mortality: A review of twenty-seven community studies. Journal of Health and Social Behavior, 38, 21-37. doi: $10.2307 / 2955359$.

Leinonen, R., Heikkinen, E., \& Jylhä, M. (2002). Changes in health, functional performance and activity predict changes in self-rated health: A 10-year follow-up study in older people. Archives of Gerontology and Geriatrics, 35(1), 79-92. doi: 10.1016/S0167-4943(02)00017-1.

Leinonen, R., Heikkinen, E., \& Jylhä, M. (2001). Predictors of decline in self-assessments of health among older people - a 5-year longitudinal study. Social Science \& Medicine, 52, 1329-1341. doi: 10.1016/ S0277-9536(00)00249-5.

Parker, M. G., \& Thorslund, M. (2007). Health trends in the elderly population: Getting better and getting worse. The Gerontologist, 47(2), 150-158. doi: 10.1093/geront/47.2.150.

Robert Koch-Institut (2015). Gesundheit in Deutschland 2015. Berlin: Robert Koch-Institut.

Robert Koch-Institut (2014). Daten und Fakten: Ergebnisse der Studie "Gesundheit in Deutschland aktuell 2012". Berlin: Robert Koch-Institut.

Robert Koch-Institut (2010). Allgemeiner Gesundheitszustand: Subjektive Gesundheit. Berlin: Robert Koch-Institut.

Schöllgen, I., Huxhold, O., Schüz, B., \& Tesch-Römer, C. (2011). Resources for health: Differential effects of optimistic self-beliefs and social support according to socioeconomic status. Health Psychology, 30(3), 326335. doi: 10.1037/a0022514.

Spuling, S. M., Wiest, M., Huxhold, O., \& Wurm, S. (2013). Bildungsabhängige Verläufe verschiedener Dimensionen der Gesundheit. In: A. Motel-Klingebiel, S. Wurm \& C. Tesch-Römer (Hrsg.) Kontexte des Alterns - Längsschnittliche Befunde des DEAS 2011. Unveröffentlichter Arbeitsbericht. Berlin: Deutsches Zentrum für Altersfragen.

UNESCO (2012). International Standard Classification of Education ISCED 2011. Montreal: UNESCO Institute for Statistics. 
170 | Subjektive Gesundheit und ihr Zusammenhang mit anderen Gesundheitsdimensionen

WHO (2002). Towards a common language for functioning, disability and health - The International Classification of Functioning, Disability and Health. Genf: WHO.
Wurm, S., Schöllgen, I., \& Tesch-Römer, C. (2010). Gesundheit. In: A. Motel-Klingebiel, S. Wurm \& C. Tesch-Römer (Hrsg.) Altern im Wandel. Befunde des Deutschen Alterssurveys (DEAS) (S. 90-117). Stuttgart: Kohlhammer.

Open Access Dieses Kapitel wird unter der Creative Commons Namensnennung 2.5 International Lizenz (http://creativecommons.org/licenses/by/2.5/deed.de) veröffentlicht, welche die Nutzung, Vervielfältigung, Bearbeitung, Verbreitung und Wiedergabe in jeglichem Medium und Format erlaubt, sofern Sie den/die ursprünglichen Autor(en) und die Quelle ordnungsgemäß nennen, einen Link zur Creative Commons Lizenz beifügen und angeben, ob Änderungen vorgenommen wurden.

Die in diesem Kapitel enthaltenen Bilder und sonstiges Drittmaterial unterliegen ebenfalls der genannten Creative Commons Lizenz, sofern sich aus der Abbildungslegende nichts anderes ergibt. Sofern das betreffende Material nicht unter der genannten Creative Commons Lizenz steht und die betreffende Handlung nicht nach gesetzlichen Vorschriften erlaubt ist, ist für die oben aufgeführten Weiterverwendungen des Materials die Einwilligung des jeweiligen Rechteinhabers einzuholen. 